\section{Passage of an Electric Discharge through Gases.}

WHEN an electric glow discharge is passed through a tube containing air at pressures of the order of 5 $\mathrm{cm}$. of mercury, the tube lying in one of the two beams in a Jamin's refractometric arrangement, there is a sudden shift of the interference fringes indicating a decrease of the refractive index of air due to the passage of the discharge. A preliminary announcement of the effect has already been made in the columns of NATURE (vol. 120, p. 880), and a detailed account of the experiments published in the Indian Journal of Physics, vol. 3, pp. 425-430.

When the discharge is stopped there is an equally sudden shift of fringes in the opposite direction and of the same extent as before, showing that the air has returned to its original state. The switching on of the discharge also causes a sudden increase in the pressure of the tube as indicated by an attached manometer, the pressure getting back to its original value as soon as the discharge is stopped. There is an exact proportionality between the fringe shift and the pressure change; and since the fringe shift if greater than one fringe width cannot be quantitatively observed on account of the suddenness of the shift, the phenomenon is better studied by observing the changes in pressure.

The investigation, which was confined to air in the above-mentioned paper, has now been extended to hydrogen, oxygen, nitrogen, carbon dioxide, and chlorine with nearly similar results as with air, namely, that for a fixed value of the applied voltage the amount of shift or the increase in pressure varies with the pressure and becomes a maximum for a certain value of the pressure, which is different for different gases and for different voltages. The effect was found to be greatest in carbon dioxide and least in hydrogen. Experiments were also made using ammonia, sulphur dioxide, and helium. In the first two the results were vitiated by the decomposition of the gas taking place under the action of the discharge, whereas in helium the effect was only just noticeable, scarcely measurable.

In all the above investigations, electrodes were, as usual, sealed into the ends of small side-tubes attached to the experimental tube, so that the electrodes were rather far away from the track of the light beam. By pure accident, however, while experimenting with air, it was found that if the electrodes are long, coming up to the periphery of the discharge tube, or rather the light beam, the fringe shift as well as the pressure change could be observed even at atmospheric pressure and when no visible glow discharge was passing. Previously, when the electrodes were fairly far away from the edge of the beam of light, the fringe shift was a maximum at a pressure of about $5 \mathrm{~cm}$. and was not appreciable at pressures below $4 \mathrm{~mm}$. or above $10 \mathrm{~cm}$. Also, in these experiments, a Tesla discharge had no effect upon the fringes. Under the new conditions, however, when the electrodes extend right up to the periphery of the light beam, both the discharge from the induction coil and the Tesla discharge produce a fringe shift even at atmospheric pressure, the effect with the latter being even greater than that with the former. The effect can thus be demonstrated very easily. It is not even necessary to close the ends of the tube to observe the fringe shift.

It is found that at atmospheric pressure the maximum effect is produced when the ends of the electrodes (which are in the form of long, stout wires or needles) are just at the periphery of the beam of light. If the end is moved into the path of the beam itself or moved away from the beam, the effect diminishes, until when it is only a centimetre away the effect disappears. Evidently, although there is no visible discharge, there is an electric field radiating from the tip of the electrode, and the effect is a maximum when the field embraces the greatest possible volume of the air. The conclusion previously arrived at, that the effect is due to a pushing away of the gas from the neighbourhood of the discharge, now receives ocular proof, for if a little lycopodium powder is dusted into the tube, it is seen that as soon as the field is put on, the lycopodium particles fly away from the electrodes.

\author{
J. B. SЕTH.
}

Bal Morand.

Physics Laboratory,

Government College, Lahore.

Properties of Dielectrics in Electric Fields.

IN NATURE of Oct. 25 is a communication from Mr. J. Mazur, under the heading of : "Change of the Dielectric Constant of Ethyl Ether with Tempera. ture ". This appears a suitable opportunity for raising a question to which I have previously alluded in several communications to the Phil. Mag. and in a long letter in NaTuRE of April 5, 1924. This is that of the nomenclature of the effects found in dielectrics in the wide range of alternating electric fields which have now for many years become increasingly available.

Let us take as a pertinent example the data given for ethyl ether in Mr. Mazur's interesting letter. An early attempt to find the dielectric properties of ether was made by Hopkinson. He first dried the ether by shaking it up with quicklime, and, using an induction coil to create the field, found $K$ was $4 \cdot 75$. Although, however, there was only a small surface of the ether exposed to the atmosphere, within a few minutes $K$ became 4.95 and thereafter observation became impossible, showing how very rapidly minute traces of moisture absorbed from the atmosphere alter the dielectric properties of matter. The above figure for $K$, and a slightly lower one due to Quincke, have long been quoted in leading tables of dielectric constants. The above experiments were at room temperatures.

Using a field of high frequency, Mr. Mazur finds for different temperatures the following results :

$$
\begin{array}{rc}
\text { Temp. } & k . \\
+30 \cdot 0^{\circ} \mathrm{C} . & 4 \cdot 18 \\
-105.4^{\circ} \mathrm{C} . & 12 \cdot 39 \\
-117.2^{\circ} \mathrm{C} . & 2 \cdot 3 \text { (melting point) } \\
-118.9^{\circ} \mathrm{C} . & 2.0
\end{array}
$$

Below this $K$ is constant and is very near $N^{2}$ for ethyl ether, which is $\mathbf{1 . 8 4}$ for the $D$ line.

In my letter mentioned above, I showed that at a range of frequencies between 8 and $2000, K$ for celluloid varied from about 12 to 6 at room temperature, and again, by intensive drying, $K$ could be made to vary from about 8 to $4 \cdot 8$ at a low frequency. Similar examples could be multiplied.

Is it not time that physicists found better names for these varying effects than calling them dielectric constants? Faraday's name, 'specific inductive capacity', was less self-contradictory. Early in the century the term 'dielectric coefficients' came into use where the property was found liable to variation, but seems to have dropped out again, possibly as being too cumbersome.

Of late years numerous connexions between dielectric and other properties have been found, and Debye's theory of molecular polarity is receiving increasing experimental support. For these reasons and others, the confusion and inappropriateness of the present nomenclature in this field, and the doubt

$$
\text { No. 3186, VoL. 126] }
$$

